\title{
Index des noms cités
}

Abastado C. 151

Adam J.-M. 82, 186, 400, 441, 445, 592, 594, 615,616

Aït-Sahlia Benaïssa, A. 471

Akesbi S. 320

Althusser L. 396, 406, 407, 411

Amorim M. 187

Amossy R. 390, 457, 467, 471, 472, 630

André C. 161

Anis J. 308

Anscombre J.-C. 42, 150, 157, 275

Anzieu D. XXVII, 495, 498, 499, 500, 502, 561, 567

Aouillé S. 408, 415

Armengaud F. 553, 570, 631

Arnaud A. 84

Arrivé M. 6, 80, 126, 131, 360, 364, 366, 463, 464

Auchlin A. 304

Aulagnier P. 508

Bair D. 603

Bakhtine M. XVIII, XXVII, 15, 129, 154, 234, 292, 376, 380-384, 386, 388, 389, 391, 392, 394, 397, 400, 419, 433, 453, 463, 482, 514

Bally Ch. 81, 83, 84, 137, 139, 317, 336, 344

Balmary M. 179

Banfield A. 105, 113, 114, 122, 127, 131, 132, $136,137,230,259,260,266$

Barthes R. 250, 343, 371, 471, 511, 513, 526, $540,565,566,570,578$

Basire B. 440

Bayard P. 150, 501

Beacco J.-C. 216

Béguelin M.-J. 208

Benveniste E. XIX-XXII, 5, 10, 117, 148, 150, $285,333,336,348,354,363,403,449$, 466, 503, 511, 545, 597

Bergounioux G. 291

Berrendonner A. 42, 157, 277, 278

Berthier Ph. 607, 627

Bloomfield L. 174

Boch F. 162, 595

De Boissieu J.-L. 313
Bolón Pedretti A. 616,617

Bonhomme M. 441

Bonnard H. 140

Bonnet C. 107

Bordas E. 174

Boré C. 80

Borges J.-L. 150

Borillo A. 77

Bouquet S. 380

Branca-Rosoff S. 152, 187, 216, 267, 457, 459

Bres J. 291, 383, 385, 386, 390, 392, 400, 415, 419, 433, 434, 435, 437, 445, 470, 479, 487, 546

Bronckart J.-P. 380, 405

Bruña-Cuevas M. 342

Brunet E. XX

Buridant C. 141

Burnier M.-A. 548

Carnap R. 250, 286, 287

Cazal Y. 192

Chabert C. 501

Chafe W. 77

Chambat-Houillon M.-F. 624

Charaudeau P. (voir aussi DAD) 416, 467, 476

Charlent M.-T. 128, 271, 273, 281

Charolles M. 74, 202, 349

Chetouani L. 470

Cislaru G. 402, 412, 458, 489, 490

Claquin F. 594

Clark H.H. 251, 357

Clavreul P. 411

Colas-Blaise M. 490, 567

Colin F. 624

Coltier D. $73,74,77$

Combettes B. 433

Compagnon A. XIV, 201, 363, 371, 441, 445, $450,451,453,564,569,627,628,630$

Conein B. 401, 403, 564

Constantin de Chanay H. 471

Courtès J. 38

Courtine J.J. 152, 396, 398, 401, 433

Culioli A. XVII, 5, 6, 9, 231, 336, 403, 435, 547, 548, 584, 597

Da Cunha D. 132, 195, 452, 593

ว Open Access. () 2020 Jacqueline Authier-Revuz, published by De Gruyter. (cc)BY-NC-ND This work is licensed under the Creative Commons Attribution-NonCommercial-NoDerivatives 4.0 License. https://doi.org/10.1515/9783110641226-026 
DAD Dictionnaire d'analyse du discours 11 , $62,360,376,377,393,440,467$

Danon-Boileau L. 348, 353

De Brabanter Ph. 287, 365

Debray R. 556, 603

De Gaulmyn M.-M. 22, 60, 305, 331, 480, 486

Delay F. 150

Delesalle S. 46

Deleuze G. 133, 150, 816, 517

Delofeu J. 342

De Mattia M. 18, 37, 84, 85, 105, 227, 236, 365

Dendale P. 76, 78

Derrida J. 150, 451, 511, 513-515, 561

Desclés J.-P. 202

Détrie C. $80,142,155,230,293,360,368$, $377,436,468$

De Vogüe S. 435

Dolto F. 495-498, 560

Doquet C. 26,584

Dor J. 409

Dosse F. 406, 414

Doury M. 481

Drillon J. 364

Dubois J. 389, 393

Dubois S. $19,40,155,340$

Ducard D. 434, 435

Ducrot 0. 9, 15, 63, 83-85, 91, 126, 275, 386, $387,417,418,420,445,471$

Dufour D.-R. 535

Dufour Ph. 211, 212

Dufourmantelle A. 495, 499, 535, 560

Dujardin E. 43

Dupriez B. 361

Ebel M. 390

Eigenmann E. 182, 520, 569, 584

Éluard P. 589

Encrevé P. 504

Fairclough N. 62

Fau F. 584

Faucher E. 126, 274

Faucher J.-M. 523, 526

Fenoglio I. XXII

Fiala P. 390, 605

Filhol E. 527, 528

Flahaut F. 411, 412, 554
Fløttum K. 307, 444

Folkart b. 587

Fontanille J. 439

Foucault M. XVII, 150, 375, 376, 386, 396, 406, 409, 415, 445

Fournier J.-M. 283

Fournier N. 13

Freud S. 327, 408, 410, 415, 500, 515

Fuchs C. 227, 336, 403

Funakoshi-Teramoto H. 439, 630

Gachet F. 256, 262, 346

Gadet F. 403, 404

Gai F. 486

Ganea A. 157

Gantheret F. 9, 501, 511, 536, 539

Gardes-Tamine J. 104

Gardin B. 389, 393, 397, 446, 469

Garnier S. 435

Gauvenet H. 37

Genette, G. 19, 131, 220, 260, 541, 569

Genevay E. XVI

Gerrig R.J. 251, 367

Godard H. 181, 630

Goffman E. 467, 595

Gollut J.D. 137, 138

Granier J.-M. 20.

Greimas A.J. 38, 237

Grésillon A. 396, 420

Grevisse M. 141, 272

Gribinski M. 494, 498, 499

Grignon 0. 415, 499, 515, 535

Grossmann F. 162, 595

Grunig B.N. 441

Guentcheva Z. 77, 79

Guespin L. 469

Guilhaumou J. 401

Guillaume G. 336, 449

Gülich E. 8

Habert B. 390

Haillet P.-P. 76, 82

Hailon F. 425, 454, 489

Hamon Ph. 222, 353, 452

Hanote S. 352

Haroche C. 396, 404

Henry P. 397, 399, 401, 402, 407, 433, 509, 510, 518

Héritier F. 188, 576, 586 
Herman Th. 630

Herschberg-Pierrot A. 18, 81, 131, 139, 230, $311,323,366,570,630$

Hjelmslev L. 248, 250

Houdebine J.-L. 411, 412, 604

Indursky F. 423

Jakobson R. 6, 8, 56, 77, 77, 174, 251, 370

Jaubert A. 22, 31, 132, 133, 136, 174, 315, $323,367,477,481$

Jeandillou J.F. 81, 131, 230, 316, 322, 363, 385

Jespersen J. 248

Jespersen 0. 62

Johansson, M. 63

Julien Ph. 411, 560

Kerbrat-Orecchioni C 39, 81, 110, 132, 153 , 202, 349, 362, 420, 437

Klein E. XVII

Klokow R. 308

Kolopp M. 609

Komur G. 306, 330, 352

Krieg /Krieg-Planque A. 179, 216, 306, 377, 390, 402, 593

Kristeva J. 380, 391

Kronning H. 75, 79

Kullmann D. 135

Kuentz P. 403, 572

Labrie N. 469

Lacan J. 6, 291, 409-411, 415, 416, 487, 494, 495, 497, 503, 504, 509, 521-523, 529, $531,536,546,565,566$

Lafont R. 469

Lagadec B. 358

Lagache D. 531

Lakoff G. 149

Laplanche J. 496

Laplantine C. XIX

Lazard, G. 77

Lecomte A. 564

Lefebvre J. 91, 349, 444, 583, 584, 627

Le Goffic P. 122, 272, 360, 363

Lejeune Ph. 311, 323, 324

Lehman A. 13

Lemoine-Luccioni E. 500, 515, 560

Lerch G. 140

Lips M. 139, 317

López-Muñoz J.-M. 369, 586
Lorda-Mur C.U. 368,391

Lucas N. 162

Lyotard J.-F. 513

Magné B. 578

Mahrer R. XX, 43, 211, 348, 349, 584, 593

Maingueneau D. XXIV, 37, 80, 81, 102, 113, 132, 152, 162, 173, 179, 185, 296, 309, $343,366,368,377,389,394,396,441$, 467, 469, 470, 471, 474, 478, 605

Maldidier D. 152, 390, 393, 394, 397-401, 404, 407, 412, 420, 584, 584

Manier A. 509, 518, 522, 527, 528

Mannoni O. 284, 409

Mansour L. 47

Marandin J.M. 397, 403, 412

Marcellesi J.-B. 390, 393, 469

Marnette S. 19, 46, 62, 82, 267, 347, 355

Martin R. 72, 126, 274, 276, 279, 280, 299, 306

Mayenova M.-R. 362

Mazière F. 394, 395, 412

Mazzuchetti D. 583, 630

Mc Dougall J. 562

Medvedev D. 380

Meiller A. 141

Mellet C. 163, 216, 349, 355

Mellet S. 435

Merleau-Ponty M. 6, 52, 512

Meunier A. XV, 22, 23, 106, 107, 127, 231, 393

Micheli R. 191, 462, 479, 614

Miller J.-A. 6

Milner J.-C. XXIII, 86, 150, 335, 362, 383, 406, 516, 521, 545, 549, 566, 580

Mochet M.-A. 18, 19, 39, 40, 42, 60, 127, 131, $142,221,267,275,276 ; 341,357$

Moeschler J. 137, 279, 417-419

Moirand S. 208, 377, 385, 389, 390, 401, 433, 434, 452, 489, 594-596

Molinié G. 167

Montaigne XIII, 147, 589

Monville-Burston M. 221

Morel M.-A. 136, 348, 353

Morgenstern A. 507

Moricheau-Airaud B. 157, 439

Mortara-Garavelli B. XV, 18, 54, 109, 132, 133, 136, 141, 363

Mortureux M.-F. 595 
Mourad G. 202, 367

Née E. 458

Neveu F. 11, 255, 360, 417

Nichols J. 77

Nicole P. 84

Nikodinoski Z. 202

Nita, R. 352

Nølke H. 18, 63, 64, 102, 103, 111, 114, 136, $142,306,311,417,418,444$

Norén C. 41, 267, 270

Normand C. 584, 585

Nowakowska A. 386, 400, 435

Ogilvie B. 409

Orlandi E. 428, 563

Ossard M. 616

Oury J. 495, 503, 567

Paillard D. 435

Paillet-Guth A.-M. 174, 236

Paveau M.A. 394, 399, 400

Pêcheux M. XVI, XIX, XXIII, XXVII, 152, 335 , $376,387,389,392-398,400-404,407$, 412, 445, 485, 512

Pernot C. 342

Perret M. 37, 81, 126, 304

Perrin L. 15, 83, 85, 262, 434, 437

Perrot M. 187

Pétillon S. 91, 124

Petiot G. 390

Peytard J. 61, 400

Philippe G. 43, 46, 132, 136, 139, 265, 311, $343,344,353,357,455,526$

Pinchon J. 14, 80, 111, 230, 272, 310

Plazaola G. I. 595

Plon M. 405, 409, 411, 412

Pommier G. 512

Pontalis J.-B. 512, 515, 534, 535, 541, 629

Porge E. 493, 497, 519, 524, 525, 531

Prieur J..-M. 469, 497, 556

Quéré H. XIV, 363, 371, 480, 486, 487, 549, 558,565

Quignard P. 504, 505, 514, 624

Quine W. van 0. 250, 287

Rabatel A. 20, 47, 64, 65, 304, 365, 385, 417, $418,420,434$

Rancière J. 576

Rannoux C. 377, 424, 574

Rastier F. 151, 152
Reboul A. 137, 279, 417, 419

Recanati F. 23, 72, 85, 204, 251, 262, 280, 287, 324

Reichler-Béguelin M.-J. 80, 116, 139, 366, 563

Rendulic N. 19, 40, 43

Rey A. 250

Rey-Debove J. 4, 9, 22, 77, 139, 203, 246, 248, 250, 251, 254, 255, 257, 258, 271, 273, 279-281, 286, 287, 289, 294, 296, $300,307,308,313,334,362,364,365$

Richard-Zapella J. 446

Riegel M. 14, 80, 105, 131, 139, 140, 229, 360,363

Riffaterre M. 186

Rinck F. 162

Roger A. 613

Romeral F. 348

Rosier L. XIV, 10, 20, 7, 43, 60, 63, 79, 81, 82, $113,143,158,267,270,271,275,280$, $305,306,320,331-334,342,348,357$, $362,365,387,394,400,415,419,481$, 602

Rosier-Catach I. 250

Rossari, C. 437

Rosset C. 466,

Roudinesco E. 408, 410, 411, 416, 490, 500, 565

Roulet E. 63

Royère A.-C. 571,572

Rubattel C. 437

Rullier-Theuret F. 363

Sadock J.-M. 126, 259

Salem A. 390

Samoyault T. 186,588

Sandré M. 624

Sapir E. 77

Sarfati G.-E. 81, 230, 272, 295, 363

Saussure F. de 254, 327, 397, 493, 512

Schepens Ph. 386, 402, 405, 522, 553, 578

Schneider M. XXV, 328, 370, 376, 378, 455, 500, 506, 511, 513, 515, 519, 520, 533-535, 542, 547, 549, 631

Schrepfer-André G. 77,81

Sériot P. 380, 392, 396, 401, 422, 433

Simonin J. 143, 184, 316, 595

Siouffi G. 512 
Sitri F. $216,349,355,390,391,402,412$, 434, 435, 458, 459, 489, 490, 583, 596. 630

Soler C. $495,501,504,505,507,509,522$, $523,525,527,528,546$

Sperber D. $188,367,440$

Starobinski J. 631

Steiner G. 149

Stolz C. 81, 126, 131, 183, 366

Strauch G. 37, 62, 113, 125, 142, 143, 202, 209

Suchet M. 468

Sullet-Nylander F. 476

Tadié J.Y. 408

Tamba I. 255, 256

Tarski A. 250

Tasmowski L. 78

Thomas J.J. 441

Tisset C. 81, 296, 363

Todorov T. 380, 382, 288

Tomassone R. 61

Torck D. 333

Tournier M. 390

Tuomarla U. 275, 276, 279, 283-285, 306, $309,331,584,593$

Turbide 0. 18

Van Raemdonck D. 36, 72, 82, 122, 260

Vanier A. 410
Vasse D. 567, 596

Velmezova E. 482

Vérine B. 435

Vernier F. 175

Verschoor J. 113

Veyne P. 457

Vincent D. 18, 19, 40, 155, 341, 471, 481

Vion R. 125

Viprey J.-M. 313

Voloshinov V.-N. XIII, 18, 35, 105, 132, 139 , $230,304,309,344,358,384,388,397$, 617

Von Münchow P. $36,41,46,50,265,278$, 306, 391-393, 604

Von Roncador M. 341

Vuillaume M. 136, 137, 318

Wagner R.-L. XXIII, 14, 80, 111, 139, 140, 230, 232, 272, 310, 360

Wahl F. 414

Wilmet M. 360

Wilson D. 367,440

Winnicott D.-W. 495, 496, 499

Wunderlich D. 46, 202, 207

Zaoui P. 452

Zourabichvili F. 517

Zufferey J. 137-139

Zwicky A. 235 